\begin{tabular}{|l|l|} 
REVISTA Revista Educación \\
ISSN: 0379-7082 \\
ISSN: 2215-2644 \\
revedu@gmail.com \\
Universidad de Costa Rica \\
Costa Rica
\end{tabular}

\title{
Aprendizaje autorregulado e intervenciones docentes en la universidad
}

[1]

Daura, Florencia Teresita

Aprendizaje autorregulado e intervenciones docentes en la universidad [1]

Revista Educación, vol. 41, núm. 2, 2017

Universidad de Costa Rica, Costa Rica

Disponible en: http://www.redalyc.org/articulo.oa?id=44051357004

DOI: http://dx.doi.org/10.15517/revedu.v41i2.21396

Esta obra está bajo una Licencia Creative Commons Atribución-NoComercial-SinDerivar 3.0 Internacional. 


\title{
Aprendizaje autorregulado e intervenciones docentes en la universidad ${ }^{[1]}$
}

\section{Self-regulated learning and instructional strategies at university level}

Florencia Teresita Daura [2]

Universidad Austral, Argentina

flodaura@hotmail.com

\author{
DOI: http://dx.doi.org/10.15517/revedu.v41i2.21396 \\ Redalyc: http://www.redalyc.org/articulo.oa?id=44051357004
}

Recepción: 06 Octubre 2015

Aprobación: 23 Junio 2017

\section{Resumen:}

Se presentan los resultados de una investigación en la que se plantearon dos objetivos: identificar las estrategias didácticas personalizadas que el profesorado utiliza en el aula y que favorecen el desarrollo de la autorregulación en sus estudiantes y elaborar un marco interpretativo que permita comprender cómo se desenvuelve la interacción académica entre el personal docente universitario y estudiantes a fin de corroborar si se manifiestan o no las estrategias mencionadas. Se instrumentó un diseño mixto, cuali-cuantitativo en el que se efectuaron observaciones no participativas de espacios de aula a cargo de 75 docentes del Ciclo Clínico de la carrera de Medicina de una universidad privada. La información tratada de forma cualitativa se analizó con la metodología propuesta por la teoría fundamentada (Strauss y Corbin, 1998) con la cual emergieron cuatro categorías de análisis, dos vinculadas con el aprendizaje autorregulado y otras dos con las estrategias mencionadas, cuya integración permitió establecer una categoría nuclear que se denominó espiral personalizante correguladora del aprendizaje. Finalmente, las subcategorías encontradas fueron cuantificadas a fin de triangular la información obtenida por el análisis cualitativo.

Palabras Clave: Autorregulación, estrategias educativas, teoría fundamentada, educación superior.

\section{Abstract:}

The present study seeks to identify the personalized classroom instruction strategies teachers use to encourage student selfregulation, as well as to construct a theoretical framework to interpret the development of the teacher student relationship at university level. A qualitative design has been adopted, with non-participant classroom observation focusing on ten subject-based courses included in the clinical course taught in Argentine medical schools. A Grounded Theory (Strauss and Corbin, 1998) analysis of the data gathered has yielded four categories: two associated with self-regulated learning and two with personalized instruction strategies. A core category has been established, which we call personalising learning co-regulating spiral. The subcategories found were quantified in order to triangulate the information obtained by the qualitative analysis.

KEYWORDS: Self-regulation, educational strategies, grounded theory, higher education.

En el nivel de la educación superior, la necesidad de un aprendizaje permanente se acentuó aún más en los últimos años recibiendo el respaldo a nivel nacional e internacional de la legislación vigente. Paralelamente, el profesorado, en forma explícita o no, manifiesta interés por conocer cuáles son las acciones que necesitan realizar para estimular en estudiantes la capacidad de "aprender a aprender"; idea que se vincula con la que Rué (2009), expresa sobre la especificidad del nivel universitario y que consiste en promover una mayor autonomía en el alumnado.

\section{NotAS DE AUTOR}

[2] Doctora en Ciencias de la educación. Disciplina de estudio: psicología de la educación. Áreas del conocimiento de interés: aprendizaje autorregulado, perspectiva temporal futura, didáctica universitaria, formación permanente, tutoría y asesoramiento académico. Líneas de investigación desarrolladas en los últimos cinco años: Estrategias didácticas personalizadas y desarrollo de aprendizaje autorregulado en estudiantado universitario; Vinculación entre la perspectiva temporal y el compromiso académico en estudiantes universitarios. Becaria doctoral CONICET (2009-2014), Becaria postdoctoral Universidad Austral-CONICET (2014-2016); docente de la Escuela de Educación de la Universidad Austral. Es coautora de: Una pedagogía del aula de mayores. Propuestas curriculares y didácticas para su desarrollo (2012), así como de dos capítulos de libros y de artículos en psicología de la educación en revistas nacionales y del extranjero (Revista Electrónica de Investigación Educativa. Forum Qualitative Social Research, Educación y Educadores, Revista de Docencia Universitaria, Revista Estudios Pedagógicos, Revista de Orientación Educacional. Revista Panamericana de Pedagogía, entre otras revistas). 
En lo que respecta al presente trabajo, el interés está puesto en conocer cuáles son las estrategias didácticas que emplea el personal docente, siguiendo un modelo de educación personalizada, para suscitar en el estudiantado universitario el aprendizaje autorregulado (AAR), capacidad que propicia el aprendizaje continuo. Esto último requiere de docentes que posean una visión y una acción estratégicas, que no se limiten a transmitir una serie de contenidos, confiando en que su eficacia reside en la cantidad de conocimientos que posean, sino que sean capaces de enseñar con autonomía sobre la base de una continua autorregulación de su accionar como enseñantes. Para lo cual, se plantean dos objetivos:

- Identificar las estrategias didácticas personalizadas que el profesorado utiliza en el aula y que favorecen el desarrollo de la autorregulación en el alumnado.

- Elaborar un marco interpretativo que permita comprender la interacción entre personal docente universitario y estudiantes.

\section{REVISIÓN TEÓRICA}

\subsection{La autorregulación del aprendizaje}

La autorregulación es una capacidad que se le exige al estudiantado universitario como un requisito para poder desenvolverse en este nivel educativo y en el medio laboral.

El interés por su estudio impulsó el desarrollo de numerosos trabajos para comprender cómo puede favorecerse en el ámbito universitario (Broc, 2011; Iglesias, Toscano y Román, 2012; Panadero y Alonso Tapia, 2014). Asimismo, se elaboraron diversos modelos teóricos sobre el AAR, entre los que se destaca el propuesto por Pintrich (2000) quien, desde una concepción sociocognitiva, lo considera como "un proceso activo de construcción por el cual se seleccionan metas académicas y se intenta monitorear, regular y controlar la cognición, la motivación, la conducta y el contexto" ( Pintrich, 2000, p.453).

Las variables que integran el AAR son (Pintrich y De Groot, 1990):

- Las estrategias cognitivas: conformadas por las estrategias metacognitivas (autorregulación), de gestión del tiempo, control del esfuerzo y administración de recursos (regulación del esfuerzo, administración del tiempo y del ambiente, búsqueda de ayuda) y las cognitivas (pensamiento crítico, elaboración, organización y repetición).

- Los componentes motivacionales de expectativas (autoeficacia, control de la tarea), de valor (metas intrínsecas, metas extrínsecas, valor de la tarea) y afectivos (sentimientos sobre sí y sobre las actividades a realizar que se expresan a través de ansiedad, vergüenza, orgullo).

Los autores Pintrich, Smith, García y McKeachie (1991) diseñaron y validaron el MSLQ (Motivated Strategies Learning Questionnaire ${ }^{[3]}$ ), instrumento que evalúa las variables mencionadas y que aquí se tomó como referencia para extraer las dos categorías que se detallan en el apartado 5 (ver Tabla 2).

\subsection{Las estrategias didácticas personalizadas}

El rasgo distintivo de la didáctica personalizada está en el vínculo docente-estudiante, en el cual el primer miembro orienta y facilita los contenidos por aprender, para que el estudiantado, como protagonista principal, los integre de modo personal (Bernardo Carrasco, 2004).

García Hoz (1988b) especificó cuatro principios metódicos con los que se sintetiza la personalización educativa y que, si se concretan en la práctica, permiten: 
- Considerar la singularidad de cada estudiante y propiciar el trabajo colaborativo, desarrollando sistemáticamente el espacio de asesoramiento académico, llamando a cada estudiante por su nombre e incentivando actividades grupales que impliquen el desarrollo de nuevos conocimientos.

- Ofrecer espacios para que el alumnado exprese qué contenidos le interesan para profundizar en relación con la asignatura.

- Propiciar la participación activa en el aula a través de actividades significativas y valiosas.

- Utilizar diversos recursos de enseñanza, entre ellos la planificación clara y flexible, que se ajuste a las características del grupo.

El mismo autor (García Hoz, 1988a) desarrolló el marco teórico que sustenta la propuesta de técnicas, métodos, procedimientos y otros recursos de enseñanza que se conciben como medios que facilitan la tarea docente y que permiten el desarrollo integral de cada estudiante (García Hoz, 1988a).

Posteriormente, discípulos y discípulas de García Hoz (1988a) (Bernardo Carrasco, 2004; Bernardo Carrasco, Javaloyes y Calderero, 2008; Rodríguez, 1996) vincularon el término de la didáctica con el de estrategia, propio del campo militar, y conceptuaron las estrategias didácticas de diversas formas, donde destaca la definición que Bernardo Carrasco (2004, p. 83) plantea como "todos aquellos enfoques y modos de actuar que hacen que el profesor dirija con pericia el aprendizaje de los alumnos .... Se [refieren] ... a todos los actos favorecedores del aprendizaje”.

En esta conceptualización, el foco está puesto específicamente en las estrategias de enseñanza y en la función de mediación que el personal docente ejerce para transmitir contenidos y favorecer que sus estudiantes aprendan, adquieran una mayor autonomía y optimicen su rendimiento académico (Daura, 2013b).

Desde esta perspectiva, se comprende que la estrategia puede estar conformada por tres elementos: método, técnicas y procedimientos. El primero está dado por la organización que se brinda a las técnicas y a los procedimientos con el fin de orientar el proceso de aprendizaje (Bernardo Carrasco, 2004); sin embargo, según nuestro entender, esta peculiaridad demuestra que el método es un concepto más abarcativo, que engloba al de estrategia y marca el camino por el cual esta tiene que diseñarse.

Por su parte, las técnicas, que no son distintas a las ya conocidas en el campo de la didáctica, se caracterizan por la selección de aquellas que propician los objetivos del proceso de enseñanza-aprendizaje. Entre ellas, García Hoz (1988) circunscribe el sistema de tutorías o de asesoramiento académico y las técnicas de grupo, que priorizan el seguimiento y el conocimiento del alumnado; no obstante, se concibe que la tutoría, más que una técnica, es una de las funciones que le corresponden al personal docente para guiar el aprendizaje del estudiantado. Asimismo, las técnicas personalizadas se complementan con el interrogatorio, el coloquio, el simposio, la mesa redonda, el panel, el torbellino de ideas, el role-playing, la exposición, entre otras.

Los procedimientos son la manera de aplicar un determinado método o forma de enseñanza desde una perspectiva predominantemente lógica (Bernardo Carrasco, 2004) para facilitar que el alumnado desarrolle un aprendizaje significativo; entre ellos se destacan el procedimiento deductivo, inductivo, simbólico, analógico, por solo mencionar algunos.

\section{Procedimientos metodológicos}

Se implementó un diseño de investigación mixto, predominantemente cualitativo, en el que los datos se analizaron con la metodología propuesta por la teoría fundamentada, también denominada teoría enraizada, emergente o grounded theory, que se fundamenta en el interaccionismo simbólico y fue desarrollada por los sociólogos Glaser y Strauss (Glaser y Strauss, 1967).

El método combina la inducción y la deducción, lo que permite relacionar estrechamente la recolección de información, el análisis y la teoría emergente (Campo y Labarca, 2009). Para ello usa distintos procedimientos 
analíticos esenciales: el planteo de preguntas; la comparación constante de incidentes; la redacción de memos y el diseño de diagramas o figuras; la codificación abierta que permite abrir categorías, subcategorías e identificar sus propiedades; la codificación axial para vincular categorías entre sí y hallar una categoría central de la que derivan las categorías y subcategorías (Strauss y Corbin, 1998, en Daura, 2014, Vasilachis de Gialdino, 2006).

Una de las riquezas propias del método es que admite integrar la metodología cualitativa y cuantitativa a partir de la vinculación de las categorías, subcategorías y categorías centrales con la información numérica obtenida.

Este tipo de análisis implica un trabajo minucioso, arduo y extenso, características que se oponen a los límites propios de un artículo científico (Gregory y Jones, 2009), por lo que en el presente trabajo se describen los procedimientos y resultados en forma sintética (Daura, 2014).

\subsection{Muestra}

Se conformó una muestra no probabilística por conveniencia (Hernández Sampieri, Fernández Collado y Baptista Lucio, 2010) integrada por 75 personas docentes titulares, adjuntas e invitadas (ver detalle en la tabla 1) del Ciclo Clínico de la carrera de Medicina de una universidad privada (UP) de la provincia de Buenos Aires (República Argentina), cuyas clases fueron observadas en forma no participativa.

La institución posee una oferta educativa de grado y de postgrado amplia, fundamentada en el movimiento de la educación personalizada (García Hoz, 1988a). A tal fin, se organizan diversos recursos que facilitan el seguimiento estudiantil (tutorías, actividades extraprogramáticas dirigidas a la integración con la vida universitaria y al desarrollo deportivo, social y humano).

En un estudio complementario al que aquí se presenta (Daura, 2014), en el que se realizaron entrevistas semiestructuradas en profundidad, se pudo saber que, si bien un grupo de docentes que forma parte del claustro académico se adhiere a esta propuesta, no todos ni todas cuentan con los conocimientos necesarios para utilizar los métodos y las técnicas personalizadas.

TABLA 1

Materias incluidas en el trabajo de campo

\begin{tabular}{|c|c|c|c|c|c|}
\hline Curso & Materia & Duración & $\begin{array}{c}\text { Horas } \\
\text { observadas }\end{array}$ & $\begin{array}{c}\text { No de } \\
\text { docentes que } \\
\text { intervinieron }\end{array}$ & $\begin{array}{c}\text { No de } \\
\text { estudiantes } \\
\text { en clase }\end{array}$ \\
\hline \multirow[t]{4}{*}{4} & $\begin{array}{l}\text { 1. Medicina Interna II } \\
\text { (MI II) }\end{array}$ & Cuatrimestral & 16 & 11 & \multirow{4}{*}{60} \\
\hline & $\begin{array}{l}\text { 2. Cuestiones de Bioéti- } \\
\text { ca (CB) }\end{array}$ & Cuatrimestral & 12 & 5 & \\
\hline & $\begin{array}{l}\text { 3. Farmacología II } \\
\text { (FARMACO II) }\end{array}$ & Cuatrimestral & 19 & 6 & \\
\hline & 4. Cirugía & Cuatrimestral & 21 & 8 & \\
\hline \multirow[t]{4}{*}{5} & $\begin{array}{l}\text { 5. Medicina Interna III } \\
\text { (MI III) }\end{array}$ & Cuatrimestral & 17 & 12 & \multirow{4}{*}{30} \\
\hline & 6. Obstetricia (OBSTE) & Cuatrimestral & 16 & 10 & \\
\hline & $\begin{array}{l}\text { 7. Ginecología (GINE- } \\
\text { CO) }\end{array}$ & Cuatrimestral & 15 & 6 & \\
\hline & 8. Pediatría & Cuatrimestral & 25 & 10 & \\
\hline \multirow[t]{3}{*}{6} & $\begin{array}{l}\text { 9. Emergentología } \\
\text { (EMERGENTO) }\end{array}$ & Bimensual & 18 & 6 & \multirow[t]{2}{*}{60} \\
\hline & $\begin{array}{l}\text { 10. Seminario de } \\
\text { Bioética (SB) }\end{array}$ & Bimensual & 10 & 1 & \\
\hline & Total & & 169 & 75 & \\
\hline
\end{tabular}

Nota: elaboración propia. 


\subsection{Instrumento de investigación seleccionado}

Se efectuaron observaciones de campo, no participativas. Para recolectar los datos, se siguieron tres pasos (Hernández Sampieri, Fernández Collado y Baptista Lucio, 1997):

A) Se definió el universo de eventos y conductas a observar. Se seleccionaron 10 asignaturas (ver tabla 1) con base en la disponibilidad y recomendación que brindaron las autoridades de la institución, en las que se observaron horas teóricas impartidas por 75 docentes (ver detalle en la tabla 1). Asimismo, antes de iniciar las observaciones, no solo el profesorado fue informado sobre la finalidad de la investigación, sino que también se solicitó su consentimiento.

B) Las observaciones se realizaron hasta que se alcanzó la "saturación teórica", esto es, cuando se comprobó que no surgían nuevos datos que enriquecieran el análisis (Strauss y Corbin, 1998).

C) Se establecieron las "unidades de observación". Aquí se optó por observar y registrar la mayor cantidad de conductas verbales y no verbales desplegadas por cada docente en el aula. A tal fin, las observaciones se registraron en forma narrativa y descriptiva, poniendo el foco en las acciones que cada docente realizaba en el aula, en los recursos y estrategias de enseñanza que utilizaba, con el fin de reflejar la concurrencia, la divergencia, la duración y la intensidad de los sucesos. A manera de ejemplificación, en el cuerpo del trabajo se detallan varios fragmentos de algunas de las observaciones realizadas; cabe aclarar que, para efectuar el análisis de los datos recopilados, se utilizó la transcripción completa de cada observación y que, dependiendo de la duración de la clase y de la dinámica que se hubiera desenvuelto en ella, tenían una extensión de tres a diez carillas con un espaciado simple.

\section{ANÁLISIS Y DISCUSIÓN DE LOS RESULTADOS}

La información recogida a través de las observaciones se transcribió en archivos para iniciar el microanálisis y el planteo de interrogantes, dos de los procedimientos propios del método de la teoría fundamentada. Ello permitió que surgieran las primeras categorías y subcategorías, que se denominaron con las diversas fuentes que sugieren Strauss y Corbin (1998): la propia imaginación de quien investiga, la terminología del marco teórico que sustenta el AAR y la didáctica personalizada y algunos códigos in vivo.

El proceso de codificación se complementó con la redacción de un guion y con el diseño de figuras o diagramas. En el guion final resultante, que se detalla en el apartado siguiente, se utilizan distintos estilos para facilitar la lectura de los elementos registrados: la fuente negrita para indicar las categorías; la fuente "itálica y entre comillas" para señalar las "subcategorías"; y la fuente itálica para resaltar las propiedades.

Se utilizó el programa Atlas.ti versión 5.0 que permitió registrar y cuantificar cuatro categorías -ver tabla 2-, dos directamente vinculadas con el AAR y dos relacionadas con las estrategias didácticas personalizadas. 
TABLA 2

Categorías y subcategorías registradas durante el análisis de las acciones docentes

\begin{tabular}{|c|c|c|}
\hline & Categoria & Subcategorias \\
\hline \multirow{9}{*}{$\begin{array}{c}\text { Categorias y } \\
\text { subcategorias } \\
\text { asociadas con } \\
\text { el AAR }\end{array}$} & $\begin{array}{l}\text { "Acciones docentes que } \\
\text { promueven la motivación }\end{array}$ & $\begin{array}{l}\text { a) Valoración de la tarea } \\
\text { b) Metas intrínsecas } \\
\text { c) Disminución de la ansiedad }\end{array}$ \\
\hline & $\begin{array}{l}\text { "Acciones docentes que } \\
\text { favorecen las estrategias } \\
\text { de aprendizaje" }\end{array}$ & $\begin{array}{l}\text { a) Elaboración } \\
\text { b) Pensamiento crítico }\end{array}$ \\
\hline & & c) Autorregulación metacognitiva \\
\hline & & d) Organización \\
\hline & & e) Aprendizaje en grupo \\
\hline & & f) Repetición \\
\hline & & g) Manejo del tiempo y del ambiente \\
\hline & & h) Regulación del esfuerzo \\
\hline & & i) Búsqueda de ayuda \\
\hline \multirow{5}{*}{$\begin{array}{c}\text { Categorias y } \\
\text { subcategorias } \\
\text { relaciona- } \\
\text { das con las } \\
\text { estrategias } \\
\text { didácticas } \\
\text { personaliza- } \\
\text { das }\end{array}$} & $\begin{array}{l}\text { "Acciones docentes que } \\
\text { facilitan la personal- } \\
\text { ización del aprendizaje" }\end{array}$ & $\begin{array}{l}\text { a) Promoción de la participación, b) Fomento del } \\
\text { pensamiento crítico, c) Rol docente facilitador, d) } \\
\text { Promoción del aprendizaje colaborativo, f) Adapta- } \\
\text { ción de las estrategias a los contenidos y al grupo, } \\
\text { g) Advertencia del saber previo del alumnado, h) } \\
\text { Interés por el grupo y por las situaciones perso- } \\
\text { nales, i) Conocimiento personal del alumnado, j) } \\
\text { Promoción de la creatividad, k) Orientación para } \\
\text { el desarrollo progresivo de la autonomia, l) Orga- } \\
\text { nización flexible del grupo, m) Incentivo de la su- } \\
\text { peración personal. }\end{array}$ \\
\hline & "Técnicas de enseñanza" & a) Técnica: Exposición \\
\hline & & b) Técnica: análisis de casos \\
\hline & & c) Técnica: Interrogatorio \\
\hline & & e) Técnica: grupo coloquial \\
\hline
\end{tabular}

Nota: elaboración propia.

\subsection{Análisis cualitativo de las observaciones}

Categoría "acciones docentes que promueven la motivación”

La denominación de esta categoría y de las subcategorías que la conforman se tomó del marco teórico elaborado por Pintrich, Smith, García y McKeachie (1991), más específicamente de los componentes motivacionales a los que hacen referencia y que se explicitan en el marco teórico previo del presente trabajo. Unas y otras expresan las acciones que docentes utilizaron para estimular la motivación del alumnado a través de la "valoración de la tarea", "las metas intrínsecas" y la "disminución de la ansiedad".

La subcategoría "valoración de la tarea" se registró en las situaciones en las que los grupos de profesores demostraron interés en el dictado de la materia y en la dedicación de tiempo para la orientación del alumnado en temas vinculados con su desempeño académico, brindando sugerencias sobre cómo tratar a pacientes, adquirir experiencia y desarrollar la mirada clínica. También, al realizar actividades de iniciación para presentar los contenidos y marcar el valor que tiene su estudio; y finalmente, cuando estimularon a sus estudiantes para que tomen conciencia del valor que tenía aprender los contenidos de la materia. Estos aspectos, que aquí se señalan con letra cursiva, durante el análisis se identificaron como propiedades de la subcategoría mencionada; el mismo procedimiento se efectuó en las siguientes subcategorías para diferenciarlas de las propiedades que las cualifican o caracterizan.

La subcategoría "metas intrínsecas" se presentó en las situaciones en las que se profundizó la explicación de contenidos para que el estudiantado los comprendiera. Esta propiedad emergió, por ejemplo, durante la 
primera clase que se observó en la asignatura Medicina Interna II, que se centró en el proceso de diagnóstico clínico y de la cual aquí se transcribe el siguiente fragmento que forma parte del momento en el que se analizaron algunos casos de pacientes; para esclarecer la ejemplificación aquí solo se destaca la subcategoría aludida y se omiten otras que también emergieron en el mismo material:

- Dr. M. (docente): Muy buena observación. En esta se aplica la Ley de Sutton. ¿Saben cuál es?

- Als (alumnos): No.

Algunos estudiantes respondieron en voz alta y otros moviendo la cabeza de izquierda a derecha, indicando que no conocían la Ley.

- Dr. M.: Sutton era un ladrón de bancos de Estados Unidos, que luego de robar varios bancos le preguntaron por qué lo hacía ahí; y respondió porque ahí estaba el dinero. Es decir, vamos a donde esté el dinero. [Como ocurre con este caso]. ... A la paciente [en la realidad] se le hizo una biopsia (el docente mostró la foto de la biopsia en una filmina) ¿De qué...? (dirigiéndose a los estudiantes). ("Metas Intrínsecas"; profundiza la explicación de contenidos para que el estudiantado los comprendan)” (Medicina Interna II, observación no participativa, clase 1, cita: 1:20)

Por su parte, la subcategoría “disminución de la ansiedad" se registró en los eventos estresantes que fueron canalizados positivamente por el personal docente, como por ejemplo, durante los exámenes en los que se generó un clima de serenidad y tranquilidad y manifestaron verbalmente su confianza en que todos obtendrían un buen rendimiento. En la asignatura Farmacología II, esta subcategoría se observó en una situación de examen en la cual la docente, si bien hizo un comentario sobre las consecuencias graves que tiene el plagio, expresó expectativas positivas sobre el desempeño positivo que tendrían todos sus estudiantes:

...Los estudiantes comenzaron a hacer silencio a medida que recibían el protocolo de examen.

- Dra. A. (docente): Me muero si le tengo que sacar una hoja a alguien. Empiecen que de seguro les va a ir bien.

(Farmacología, observación no participativa, clase 5, cita 5:15)

A medida que se fueron registrando estas subcategorías, se comprobó que coincidían con otras que integran la categoría acciones que facilitan la personalización del aprendizaje. Particularmente, ello ocurrió con "valoración de la tarea", ya que cuando el profesorado la promovió, se produjo una mayor interacción entre docente y estudiantes y, consecuentemente, una mayor participación en clase. Un ejemplo puede verse en el siguiente fragmento de la segunda clase observada en la materia Emergentología, que se focalizó en el estudio del trauma y en el cual se produjo una gran participación por parte del estudiantado a partir de las intervenciones docentes, principalmente dirigidas a explicar el contenido y orientar el proceso de enseñanzaaprendizaje:

El doc. 1 (docente 1) abrió una página en internet (www.trauma.org/resus/moulage/moulageone.html <http://www.trauma.org/resus/moulage/moulageone.html> y pidió que alguien leyera la información que estaba en inglés.

- Doc. 1: ¿Alguno que lea bien en inglés?

Un alumno se ofreció y comenzó a leer los datos del caso, que estaba diseñado de tal forma que en un momento debía elegirse una de varias opciones a seguir.

Al llegar a ese punto, entre todos los presentes comenzaron a elegir la acción correcta.

El doc. 2 (docente 2) continuó de pie al lado de la computadora y desde allí iba seleccionando con el mouse la opción indicada por los alumnos. En un momento se detuvo para explicar una acción concreta que se planteaba en el caso. De esta forma, entre todos iban eligiendo qué acciones debían efectuar con el paciente del caso. La página estaba diseñada de forma tal que, si una acción era errónea, se visualizaba un cartel que así lo indicaba. ("Valoración de la tarea", Muestra interés en el dictado de su materia y en la dedicación de tiempo para la orientación del alumnado)

El doc. 2 continuó explicando cuáles son las acciones correctas a llevar a cabo en cada caso. A las 17,11 horas ingresó al aula otro docente que integraba la catedra. ("Valoración de la tarea", Muestra interés en el dictado de su materia y en la dedicación de tiempo para la orientación del alumnado) 
Entre todos continuaron analizando el caso. En un momento, el doc. 3 (docente 3 ) indicó a los alumnos:

- Doc. 3: Fíjense cómo le varió la presión arterial del paciente.

Esta información figuraba en el vértice izquierdo superior de la pantalla. Los estudiantes expresaron su asombro y se rieron por no haber visto antes estos datos ... ("Fomento del pensamiento crítico", "Promoción de la participación", "Promoción de la creatividad", "Promoción del aprendizaje colaborativo") (Emergentología, observación no participativa, clase 2).

Un efecto similar se produjo entre las subcategorías motivacionales y algunas que integran las acciones que favorecen las estrategias de aprendizaje. Específicamente cuando el profesorado, al tiempo que profundiza algún contenido (propiedad de la subcategoría metas intrínsecas), favoreció la "repetición", la "elaboración", la "búsqueda de ayuda", el "aprendizaje en grupo" y el "pensamiento crítico".

Según las subcategorías registradas en la categoría acciones docentes que promueven la motivación, las propiedades que se reconocieron en cada una de ellas, la frecuencia con la que se presentaron y las interacciones observadas, se comprobó que los grupos docentes no estarían muy preocupados en suscitar este aspecto del proceso de enseñanza-aprendizaje, no saben cómo hacerlo o lo consideran innecesario por la etapa de la carrera en la que el estudiantado se encuentra.

Categoría "acciones docentes que favorecen las estrategias de aprendizaje"

Esta categoría se refiere a las acciones que docentes realizaron para estimular las estrategias de aprendizaje en el alumnado; en ella se identificaron varias subcategorías cuya denominación se apoya en el marco teórico expuesto (Pintrich, Smith, García y Mc Keachie, 1991).

La subcategoría "elaboración", se registró con una mayor frecuencia, cuando el personal docente facilitó la organización del conocimiento por medio del uso de diversos organizadores gráficos, propiedad que coincidió con otra de la subcategoría "organización", en las ocasiones en las que se utilizaron estos recursos para secuenciar los temas dictados en clase y ordenar el propio discurso.

La "elaboración" del pensamiento también se promovió cuando se establecieron relaciones entre los contenidos propios de la asignatura, y entre esta y otros espacios curriculares. En esta subcategoría se encontraron otras dos propiedades: incentivar al alumnado para que estudiara reuniendo información de distintas fuentes bibliográficas y favorecer la integración de conocimientos. Esta última propiedad se presentó fundamentalmente en los exámenes que se observaron en Farmacología II, Medicina Interna III, Ginecología, Obstetricia y Pediatría; también durante las horas prácticas de Farmacología II en las que se propusieron actividades que implicaron la reformulación personal de los contenidos; y en las clases teóricas en las que se utilizó la "técnica del interrogatorio" con el objeto de vincular los conocimientos previos con los nuevos. El fragmento que se presenta a continuación permite ilustrar cómo se presentó esta propiedad en una clase de la asignatura Medicina Interna II, a partir de la exposición y de las preguntas formuladas por el docente, aquí vale aclarar que, si bien durante el análisis de este fragmento también se registraron otras subcategorías, solo se resalta la subcategoría "elaboración":

El docente, para dinamizar la participación de los alumnos, les preguntó qué cosas comúnmente manifiestan los pacientes sobre la sintomatología que padecen.

- Dr. H.: ¿Cómo y qué le preguntarían al paciente?

Los estudiantes comenzaron a expresar sus opiniones con mucha animosidad, mientras se reían. Ante el comentario de una alumna, el Dr. H. acotó:

- Dr. H.: Sean creativos. Muy bien lo que ella dijo, hace referencia a la progresividad (...).

Allí marcó la importancia que tiene la formulación correcta de las preguntas diagnósticas, a lo cual lo ejemplificó con casos reales.

- Dr. H.: ¿Qué estudios harían?

Los estudiantes respondieron con ayuda del docente, el cual aportaba contenidos teóricos.

- Dr. H.: ¿Qué otros estudios harían?

Los alumnos continuaron opinando. 
- Dr. H.: Bien... placas...; un ecocardiograma ¿Les parece importante?

- Als.: Si.

- Dr. H.: Chicos es EL estudio importante.

A su expresión el Dr. H. la apoyo con algunas anotaciones en el pizarrón. (Medicina Interna II, observación no participativa, clase 2).

En algunas oportunidades, la "elaboración" coincidió con otras subcategorías de las acciones que favorecen las estrategias de aprendizaje; en concreto, con el "aprendizaje en grupo" cuando se dedicaba tiempo para discutir entre todo el alumnado presente alguna temática y para formular preguntas dirigidas a la reflexión y a la integración de lo tratado. Del mismo modo, coincidió con la subcategoría "pensamiento crítico" en las situaciones en las que se abrieron espacios para intercambiar ideas sobre algún tema y se solicitó al estudiantado que expresara sus opiniones, o bien se realizaron comparaciones para encontrar las semejanzas y las diferencias entre los contenidos estudiados, suscitándose la integración de conocimientos. Convergencias similares se encontraron con la subcategoría "búsqueda de ayuda" cuando el profesorado se mostró disponible para responder las preguntas formuladas por sus estudiantes y planteó interrogantes para incentivar la elaboración cognitiva. También con la "autorregulación metacognitiva" cuando preguntó a sus estudiantes si habían comprendido los temas dictados y distinguieron las ideas importantes de aquellas que no lo eran.

En segundo orden de frecuencia, por el número de veces en que se registró, se encuentra la subcategoría "pensamiento crítico" en la que se identificaron las siguientes propiedades: apertura de espacios para intercambiar ideas sobre un tema; solicitar a estudiantes que argumenten sus ideas o formulen preguntas; suscitar comparaciones para encontrar semejanzas y diferencias entre los contenidos. Por sus mismas características, se presentó en los momentos en los que se utilizaron las técnicas "análisis de casos", "interrogatorio" o el "grupo coloquial” y durante la "exposición”. También coincidió con otras subcategorías: "aprendizaje en grupo", cuando se abrieron espacios para intercambiar ideas, y "organización” de los contenidos.

En tercer orden de frecuencia se presentó la subcategoría “autorregulación metacognitiva” en las oportunidades en las que el personal docente impulsó el control de la atención de estudiantes, distinguió las ideas importantes de las que no lo eran, efectuó indicaciones sobre la bibliografía a utilizar y corroboró la comprensión de los temas dictados, por ejemplo, con preguntas similares a esta "¿Me comprendieron?" o “¿Vamos bien hasta acá?”. Abundantes ejemplos de esta subcategoría se encontraron en la asignatura Emergentología, como el siguiente:

El Docente 1 (Doc. 1) ingresó nuevamente al aula y dijo:

- Doc. 1: ¿̨esolvemos el último caso?

Una alumna leyó el caso y entre todos empezaron a analizarlo. Cuando lo resolvieron el Doc. 1 dijo que lo que querían era que los estudiantes se dieran cuenta que muchas decisiones se toman en equipo.

A las 17,53 horas el Doc. 1 dijo:

- Doc. 1: Eso es todo chicos. Hasta el miércoles. (Emergentología, observación no participativa, clase 2).

El registro de esta subcategoría aumentó cuando el personal docente titular o adjunto intervino con más asiduidad. No obstante, las propiedades que se presentaron en esta muestran que solo se estimuló para favorecer la comprensión de las acciones que el profesorado realizó en el aula y no para estimular un mayor control de la cognición de sus estudiantes; esto como consecuencia de que las intervenciones de docentes se dirigieron a preguntar a si comprendían la explicación que estaban dando sobre el tema central de la clase. Este efecto también se asocia con las técnicas de enseñanza que se utilizaron mayoritariamente y que permitieron que la "autorregulación metacognitiva" se presentara conjuntamente con la subcategoría "técnica: exposición”. 
En el cuarto orden de frecuencia se registró la subcategoría "organización”, en la que se identificaron tres propiedades: utilizar recursos gráficos para secuenciar los temas dictados, presentar los objetivos de la asignatura o de la clase para guiar el aprendizaje; advertir que la clase está inserta en un plan de trabajo -por la introducción del tema o la referencia a temas previos o próximos.

La subcategoría "aprendizaje en grupo", por su parte, se registró en quinto orden de frecuencia, cuando el personal docente promovió el trabajo colaborativo utilizando distintos tipos de técnicas, inclusive durante la "exposición". De esta manera, en Cirugía y Pediatría, el "aprendizaje en grupo", se favoreció a través del desarrollo de los talleres o de coloquios en los que se profundizaron conocimientos sobre los casos vistos durante las horas prácticas. En Farmacología II, los trabajos prácticos que se realizaron durante las últimas horas de clase, también propiciaron la presencia de esta subcategoría.

Con una mínima frecuencia se presentaron las subcategorías "repetición", "manejo del tiempo y del ambiente", "regulación del esfuerzo" y "búsqueda de ayuda". Entre estas, la "regulación del esfuerzo" se registró en los momentos en los cuales el personal docente marcó los distintos momentos de la clase (inicio, recreos, etc.), caso como el que se presentó en una de las clases de Emergentología:

Luego de analizar un caso el doc. 1 (docente 1) expresó:

- Doc. 1: Bueno, nos quedan nada más que la $\mathrm{C}$ y la $\mathrm{D}$ y pasamos al práctico. Pueden ir a tomar un café y vuelven (...) en 15 minutos. (Emergentología, observación no participativa, clase 3 ).

Categoría "acciones docentes que facilitan la personalización del aprendizaje"

Esta categoría refiere a las acciones que el personal docente realizó con el fin de facilitar un proceso personalizado tendiente a desarrollar todos los aspectos que integran a la persona estudiante.

La subcategoría "promoción de la participación" se destacó por ser la que tuvo un mayor número de registros, en los momentos en los que se propició la intervención activa de estudiantes, el intercambio de ideas sobre una temática en particular. Ello se produjo principalmente a través de la utilización de distintas técnicas de enseñanza como la "exposición"; la "formulación de preguntas" "interrogatorio", el "análisis de casos" o el "grupo coloquial" con las que se propuso realizar diversas actividades en el aula. A medida que se registró y se produjo la saturación teórica, su presencia coincidió con otras subcategorías de las categorías ya mencionadas (como las "metas intrínsecas", la "elaboración”, el "aprendizaje en grupo") y de las "acciones que facilitan la personalización", particularmente con "la promoción del aprendizaje colaborativo".

En orden seguido, se presentó la subcategoría "fomento del pensamiento crítico", efecto que concuerda con uno de los aspectos que tratan de incentivarse en el contexto de la educación personalizada: el desarrollo de la capacidad crítica. Por sus particularidades, convergió con la subcategoría "pensamiento crítico" que forma parte de las acciones que favorecen las estrategias de aprendizaje, con la que comparte dos propiedades: abrir espacios para intercambiar ideas y solicitar a estudiantes que argumenten sus ideas o formulen preguntas. A fin de ilustrarlo, se presenta un fragmento de la primera clase observada en la asignatura Seminario de Bioética en la cual se analizó un caso:

...La dinámica en la clase continuó desarrollándose de la misma forma, los estudiantes participaban activamente expresando sus conocimientos previos e ideas personales (sobre el caso que se estaba analizando de incumbencia ética), dando lugar al aprendizaje colaborativo; paralelamente, el docente actuaba como guía y orientador de la actividad, nombrando a cada alumno por su nombre.

- Docente: (...) ¿A ver quién discute la posición de Federico?

- Algunos alumnos respondieron en forma ordenada, respetando la palabra del otro compañero. (Seminario de Bioética, observación no participativa, clase 1).

Estas dos subcategorías también se estimularon a través de la respuesta de preguntas y de la formulación de interrogantes, ante los cuales se abrió la subcategoría "rol docente facilitador" cuya propiedad principal fue demostrar cercanía hacia el estudiantado, y que se caracteriza por ser un soporte del proceso de enseñanza- 
aprendizaje, por estar disponible para responder las inquietudes del alumnado referidas a la materia o a la carrera y por favorecer sus iniciativas, aspecto vinculado con la proactividad.

En quinto orden de frecuencia se registraron las subcategorías "adaptación de las estrategias a los contenidos y al grupo", cuando el profesorado indagó sobre los temas que se habían estudiado durante las clases anteriores, y la "advertencia del saber previo del alumnado" cuyas propiedades son: la interrogación, el reconocimiento personal y la promoción de la participación. De hecho, la primera subcategoría emergió en el siguiente fragmento de una de las clases observadas en Emergentología, en la cual el docente anticipó el tema central que abordaría:

La clase inició puntualmente a las 15,00 horas. En el aula estaba el Doc. 1 e informó que iban a tratar tres temas importantes, entre ellos trauma de cráneo.

El docente preguntó a los alumnos si en neurología habían estudiado el tema y entendían lo que era el cuadro; los estudiantes le informaron que lo habían abordado el año anterior, por lo que el profesor comenzó a detallar los objetivos de la clase. (Emergentología, observación no participativa, clase 3 ).

Las intervenciones docentes a través del interrogatorio favorecieron la apertura de una nueva subcategoría a la que se denominó “interés por el grupo y por situaciones personales". Esta no solo incentivó la participación del alumnado, sino que también responde a una de las características propias de la personalización educativa, que es la preocupación sincera por el proceso de aprendizaje estudiantil.

La misma subcategoría está en vinculación con la que se reconoció como "conocimiento personal del alumnado que se presentó en séptimo orden de frecuencia, en las ocasiones en las que el personal docente demostró conocer y llamar por sus nombres o apellidos a sus estudiantes por haber compartido otros espacios de aprendizaje -por ejemplo, las horas prácticas desarrolladas en los hospitales-; y en las que promovió la participación, propiedad que fue más frecuente ante la presencia de docentes titulares.

La "promoción de la creatividad" se reconoció en aquellas situaciones en las que el profesorado, por medio del planteo de preguntas sobre el marco teórico o un caso clínico, suscitó la expresión de ideas originales y apropiadas por parte de sus estudiantes. Por lo general, se presentó en los momentos en los que se promovió el "aprendizaje colaborativo" y "la participación”.

En noveno orden de frecuencia se registró una subcategoría, interesante por las propiedades que la representan, y que se reconoció como "orientación para el desarrollo progresivo de la autonomía”. Esta se presentó en las ocasiones en las que el personal docente expresó su preocupación por el desempeño académico y profesional de sus estudiantes o se mostró dispuesto a brindar una guía en ambos aspectos. Esta subcategoría coincidió con algunos códigos "in vivo" tomados del discurso de docentes que integraban la cátedra de Cirugía y que demuestran su interés por guiar el proceso de aprendizaje del alumnado:

Ustedes han estudiado hasta ahora de una forma, (...) ¡Señores! ¡Esto se terminó! Ustedes no estudiarán para ser alumnos, sino que estudiarán para ser médicos. Aprender es aprender conductas y ahora tienen que cambiar las que ya venían haciendo" (Cirugía, observación no participativa, clase 1).

La "organización flexible del grupo" se utilizó para representar las situaciones en las que el profesorado indicó al alumnado que efectuara exposiciones didácticas, trabajos en equipo o individuales. Esta se registró en las materias Cirugía y Farmacología durante las actividades prácticas.

Por último, se abrió la subcategoría "incentivo de la superación personal" que se presentó cuando el personal docente, a través de expresiones verbales, consideró las posibilidades de mejora del alumnado.

La categoría nuclear del análisis "espiral personalizante correguladora del aprendizaje"

A medida que se continuó con el análisis de los datos, se comprobó que el personal docente promovió "la personalización", la "motivación" y las "estrategias de aprendizaje" pero solo accidentalmente, pues su principal interés fue la transmisión de contenidos y el desarrollo de su comprensión por parte del alumnado.

También se evidenció que las ocasiones en las que se promovió la personalización del aprendizaje coincidieron con el desenvolvimiento de la motivación y de las estrategias de aprendizaje en el alumnado; punto en el cual se pudo abrir y establecer la categoría central o nuclear del análisis, paso necesario para 
integrar la teoría (Strauss y Corbin, 1998, p.102) y que representa el tema central de la investigación en torno al cual las demás categorías se vinculan: espiral personalizante correguladora del aprendizaje (ver figura 1). A partir de ello, se consideró que la personalización es el núcleo que permitiría el desarrollo de los factores que intervienen en el AAR (Daura, 2013a).

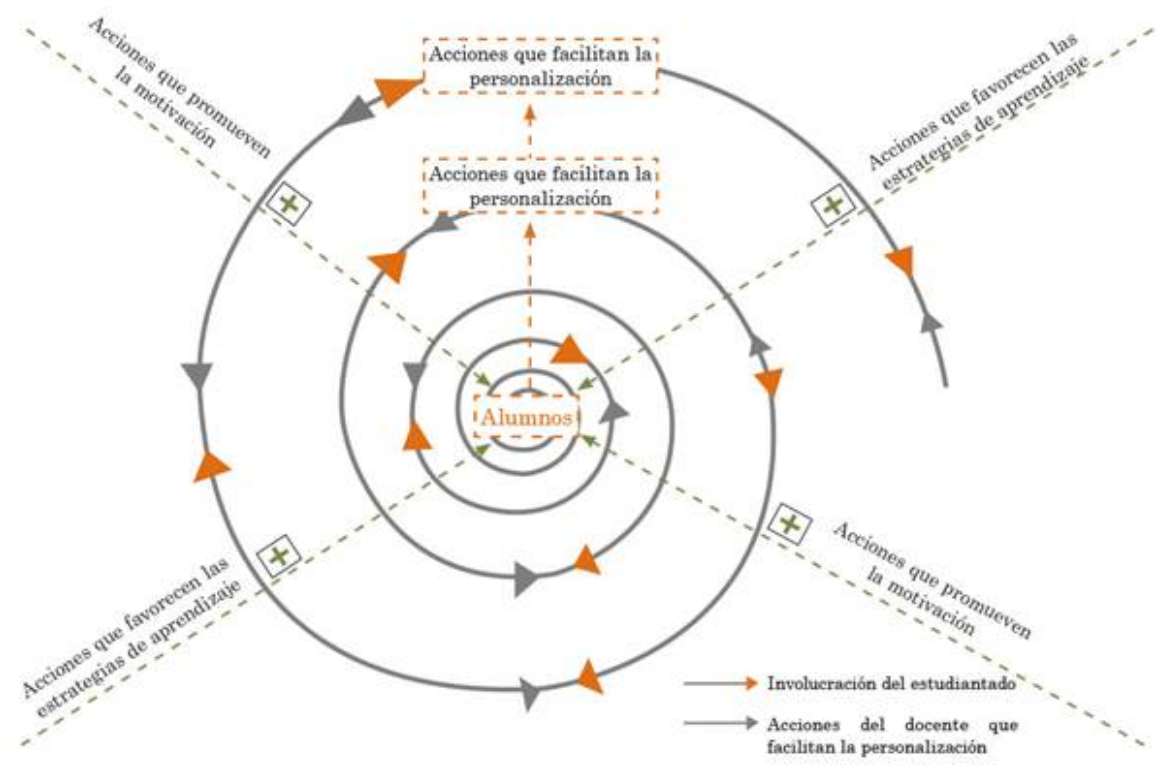

FIGURA 1

La categoría nuclear del análisis "espiral personalizante correguladora del aprendizaje Daura 2013b, p. 271

En efecto, el registro de alguna subcategoría que integra la categoría acciones que facilitan la personalización siempre coincidía con otras que forman parte de las acciones que promueven la motivación y las acciones que favorecen las estrategias de aprendizaje. Según esta idea, en la medida en que el personal docente incentivó la participación, el conocimiento personal de cada estudiante, manifestó predisposición por saber cómo se llamaban, entre otras actitudes vinculadas con la categoría acciones que facilitan la personalización, el estudiantado demostró una actitud más activa e involucrada hacia su aprendizaje, lo que, a su vez, repercutió positivamente en el profesorado que continuó favoreciendo la participación, el interés por el grupo clase, aumentando así las oportunidades para que se desarrollaran la motivación y las estrategias de aprendizaje (Daura, 2013a), variables que conforman la capacidad autorreguladora. No obstante, esto no fue una generalidad, ya que no se presentó intencionalmente en las clases observadas, sino que se dedujo a partir del análisis de los datos que emergieron en las observaciones; por lo que puede considerarse que, en el caso de que el personal docente persiguiera estratégicamente este fin -personalizar el proceso de enseñanzaaprendizaje para motivar y desplegar estrategias de aprendizaje, ello incidiría positivamente en el desarrollo de mayores niveles de autorregulación en sus estudiantes.

Sobre estos hallazgos, una cuestión interesante a resaltar es que coinciden con los encontrados en trabajos previos (Daura, 2013a; Daura, 2013b), aspecto que permite confirmar la teoría resultante con la utilización del método de la teoría fundamentada.

\subsection{Análisis cuantitativo de las observaciones}

Las categorías y subcategorías registradas fueron cuantificadas a fin de corroborar la información obtenida en el análisis cualitativo. A partir de ello, se calculó la "tasa" o relación existente entre los valores obtenidos en cada subcategoría con el número total de horas observadas en todas las asignaturas ${ }^{[4]}$. 
En la Figura 2 se presentan las tasas correspondientes a las subcategorías que conforman las acciones docentes que promueven la motivación. En el mismo puede comprobarse que la subcategoría "Valoración de la tarea” alcanzó la mayor tasa en comparación con las otras dos subcategorías, por lo que se refuerza la idea expresada en el análisis cualitativo del presente trabajo según la cual, esta subcategoría fue la más incentivada por el personal docente en las clases observadas.

Según la prueba de diferencia de proporciones, la desigualdad encontrada entre el mayor y el menor valor, que corresponde a las subcategorías valoración de la tarea y disminución de la ansiedad, no es estadísticamente significativa $(Z=1,23)$.

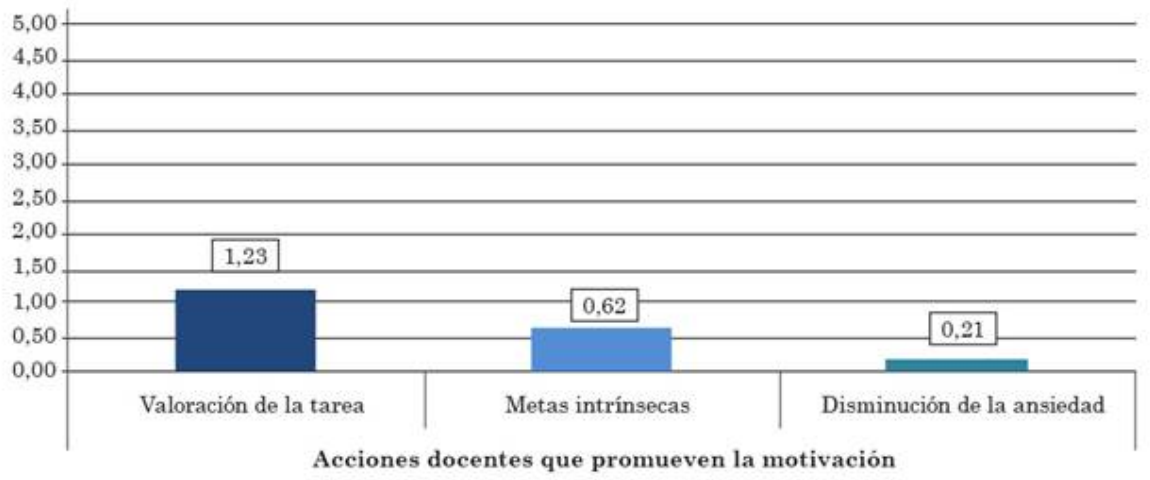

FIGURA 2.

Tasas de las subcategorías que integran la categoría "acciones docentes que promueven la motivación Elaboración propia

Las subcategorías que integran las acciones docentes que favorecen las estrategias de aprendizaje se representan en la Figura 3. En este caso, entre elaboración y regulación del esfuerzo, que son las subcategorías que poseen el mayor y el menor valor respectivamente, tampoco se halla una diferencia estadísticamente significativa $(Z=1,54)$; por lo que, si bien la primera emergió notoriamente en el análisis, su presencia no marcaría diferencias fundamentales en comparación con la subcategoría regulación del esfuerzo.

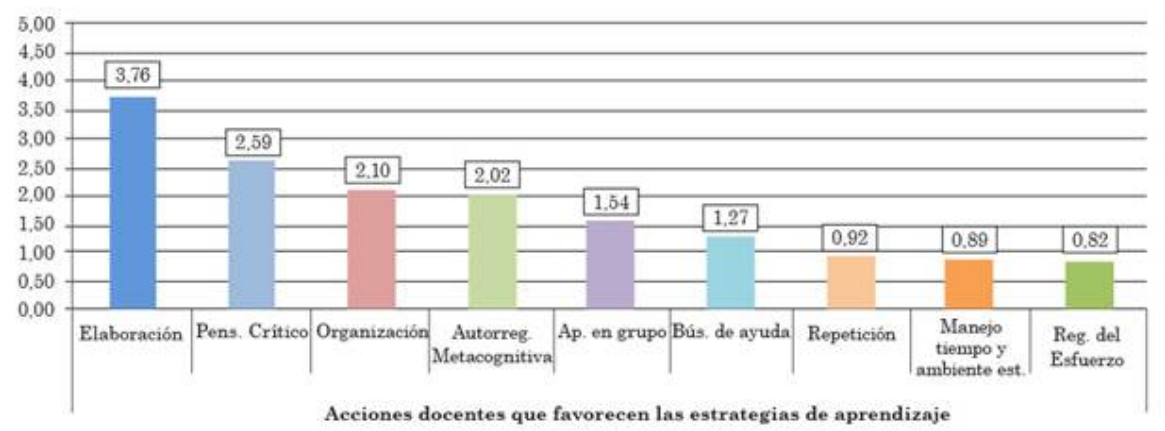

FIGURA 3

Tasas de las subcategorías que integran la categoría "acciones docentes que favorecen las estrategias de aprendizaje” Elaboración propia

Las subcategorías que forman parte de las acciones docentes que facilitan la personalización del aprendizaje se representan en la Figura 4; caso en el cual la promoción de la participación y el incentivo de la superación personal alcanzaron una diferencia significativa $(Z=2,22, \mathrm{p}<0,05)$. 


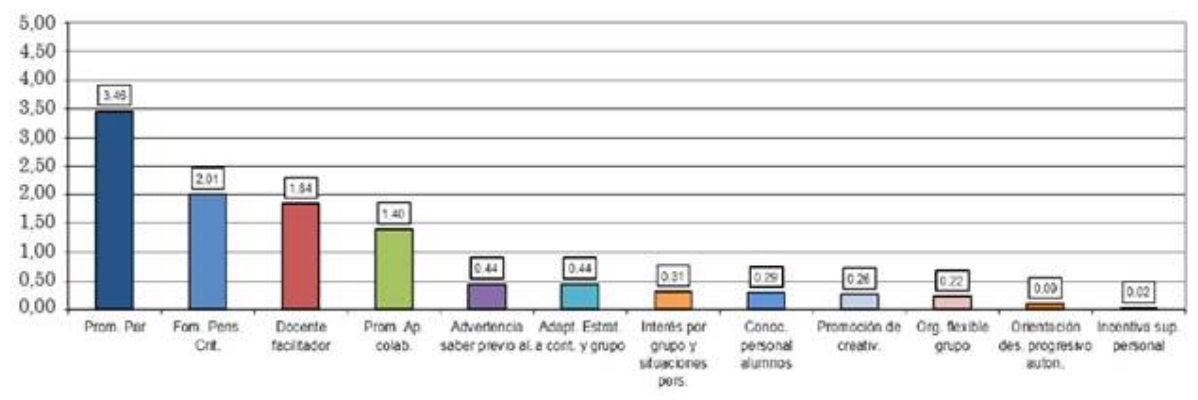

FIGURA 4.

Tasas de las subcategorías que conforman las "acciones docentes que facilitan la personalización del aprendizaje"

Elaboración propia

Es de destacar que el mismo análisis efectuado sobre el valor alcanzado por el total de las subcategorías que integran cada una de las categorías principales (ver Figura 5), arrojó diferencias estadísticamente significativas que corroboran el análisis cualitativo. Particularmente, entre las acciones que promueven la motivación y las que favorecen las estrategias de aprendizaje, a favor de estas últimas $(Z=4,41, p<0,05)$, efecto que permite sustentar los hallazgos del análisis cualitativo con los que se expresó que el personal docente promueve más las estrategias cognitivas o de aprendizaje, en detrimento de las variables motivacionales.

Del mismo modo, se encontraron diferencias estadísticamente significativas entre las que acciones que promueven la motivación y las que facilitan la personalización del aprendizaje, en detrimento de las primeras $(\mathrm{Z}=2,89 \mathrm{p}<0,05)$.

También se encontraron diferencias significativas a favor de las acciones docentes que favorecen las estrategias de aprendizaje $(Z=4,06, p<0,05)$ y de las acciones del profesorado que facilitan la personalización $(\mathrm{Z}=2,57, \mathrm{p}<0,05)$ en relación con las técnicas de enseñanza.

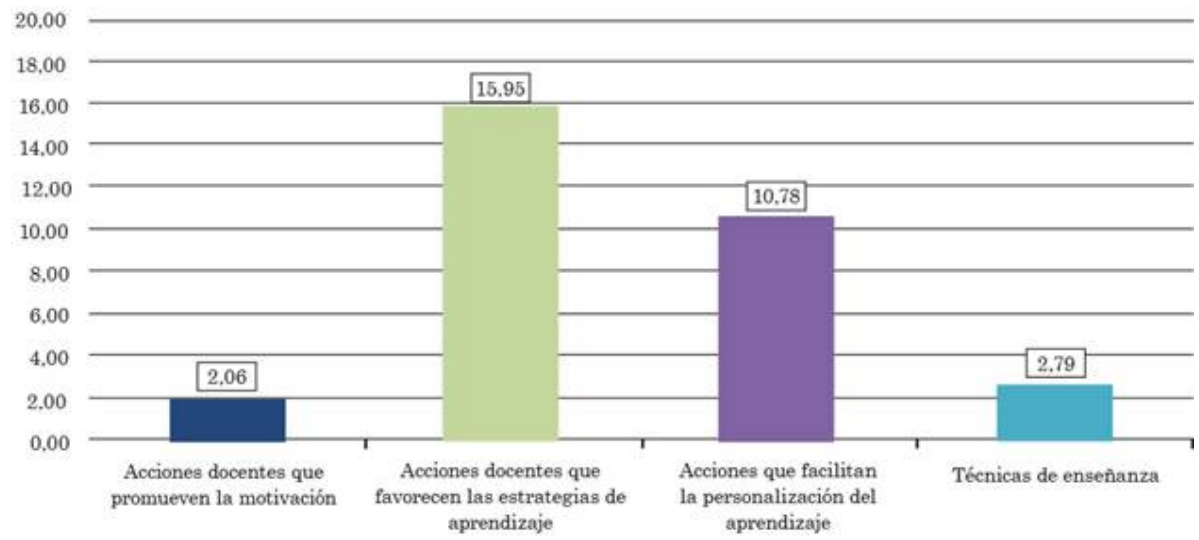

FIGURA 5.

Comparación de las tasas obtenidas por las cuatro categorías principales extraídas en el análisis. Elaboración propia.

\section{Conclusiones}

La autorregulación es una capacidad que todo el estudiantado universitario tiene que desarrollar con la finalidad de adaptarse a las exigencias propias del nivel educativo en el que se encuentra y del futuro mundo laboral en el que tendrá que insertarse. 
Por ser factible de desarrollarse a lo largo de toda la vida, queda atrás la idea con la cual se sostenía que su adquisición era innata o hereditaria y que, por tanto, al ingresar a un estudio superior, se estaba en condiciones de desenvolver las estrategias que el AAR conlleva. Ello exige un rol docente más activo, con mayor implicación en el proceso de enseñanza-aprendizaje.

La didáctica personalizada, asimismo, muy declamada y poco puesta en práctica sobre todo en el ámbito universitario, tal vez por su identificación con la individualización del aprendizaje, ofrece recursos que pueden ser fácilmente utilizados en este nivel. Puede considerarse que, si se implementa correctamente, podría propiciar la autorregulación del aprendizaje al tener por finalidad el desarrollo integral del estudiantado.

En relación con estos constructos, en el presente estudio, de carácter mixto y eminentemente cualitativo, se plantearon dos objetivos, para cuyo cumplimiento se efectuaron observaciones no participativas con las que se recogieron datos que fueron analizados con el método propuesto por la teoría fundamentada (Strauss y Corbin, 1998).

A partir de este último, se identificaron cuatro categorías de análisis, dos vinculadas con el AAR -acciones que promueven la motivación y acciones que favorecen las estrategias de aprendizaje- y otras dos asociadas con la didáctica personalizada -acciones que facilitan la personalización del aprendizaje y técnicas de enseñanza-. La utilización de la codificación axial, uno de los procedimientos característicos del método, permitió vincular estas categorías y obtener una categoría central que representa el marco teórico enraizado. A la misma se la denominó "espiral personalizante correguladora del aprendizaje” y se registró al comprobar que, en la medida en que enseñantes promovían un proceso educativo personalizado, caracterizado por la cercanía con sus estudiantes y por el interés en su desarrollo integral, entre otros aspectos, se presentaban más oportunidades en las que se favorecían las variables que integran el AAR.

La información cualitativa se complementó con el análisis cuantitativo que permitió corroborar las ideas y, al decir de Hernández Sampieri, Fernández Collado y Baptista Lucio (2010, p. 546) obtener una "fotografía" [5] más completa del fenómeno de estudio".

Estos efectos, en relación con los planteos previos, obligan, por un lado, a reflexionar sobre el rol y la función docente en la universidad, como responsable de promover la construcción de saberes, brindar un modelo profesional y personal, y orientar el proceso de enseñanza-aprendizaje. Por el otro, muestran la necesidad de organizar el proceso educativo de manera personalizada en pos de desarrollar todas las potencialidades de cada estudiante; más aún el de quien transita el nivel universitario, caracterizado en algunas oportunidades por la masividad y la multiculturalidad, aspectos que provocan el aumento de la despersonalización y la disminución de la motivación por aprender.

Sin duda, ello presenta un reto para quienes en la actualidad se desempeñan como profesionales de la enseñanza, ya que personalizar la educación requiere de una mayor dedicación para conocer a cada estudiante en particular, evaluar el propio accionar docente y modificar aquello que sea necesario para suscitar profesionales capaces de aprender a aprender a lo largo de toda la vida.

En línea con estas ideas se ubica el diseño y la implementación de estrategias didácticas personalizadas, entre las cuales no solo se destaca la tutoría, sino también otras que favorecen el desarrollo del pensamiento crítico y reflexivo, como lo son aquellas que forman parte del aprendizaje basado en problemas (ABP) (DíazBarriga, 2009; Huber, 2008); o las que se dirigen a desarrollar la motivación, entre las que se distinguen la promoción de la participación activa del alumnado, el interrogatorio y el interés por las necesidades estudiantiles.

La utilización de estas estrategias implica volver a lo que Pérez (2012, p. 11) denomina "la raíz originaria de la educación" la cual se encuentra en la relación que entablan quien educa y estudiantado, y que consiste en un enriquecimiento mutuo, en el que el primer sujeto transmite su saber y se nutre de lo que aprende del segundo, mientras que este se forma integralmente a partir del modelo recibido. En efecto, si bien estas ideas se enmarcan dentro del aprendizaje permanente, el rol y la función docente no dejan de ocupar un lugar esencial, por ser quien, por el solo hecho de tener una mayor trayectoria de vida, puede transmitir los 
aprendizajes logrados y las estrategias que utilizó para alcanzarlos; efectivamente, la utilización de la estrategia de modelado (Bandura, 2000, 2009), es la que da la posibilidad de mostrar con acciones concretas cómo se efectúa una tarea o actividad, cuáles son los pasos que se realizaron para elaborar un razonamiento o un cálculo y cómo se evalúa el propio desempeño en el proceso de aprendizaje (Boekaerts y Corno, 2005; Pintrich, 2002; Zimmerman, 1989).

En pos de ello, los recursos de enseñanza tienen que considerarse como medios que coadyuvan a alcanzar el fin del proceso educativo y que pueden utilizarse en el espacio áulico para posibilitar el desarrollo intelectual, afectivo-volitivo, cultural y trascendente del estudiantado, en vistas a promover su autonomía.

En el ámbito universitario pueden ofrecerse programas de desarrollo docente con los que se adquieran conocimientos pedagógico-didácticos dirigidos a mejorar la enseñanza, de manera que el profesorado no se limite a transmitir conocimientos teóricos, sino que también estimule todas las capacidades estudiantiles y procure fomentar en este ser una mayor autorregulación.

\section{REFERENCIAS}

Bandura, A. (2000). Exercise of Human Agency Through Collective Efficacy. Current Directions in Psychological Science, 9(3), 75-78.

Bandura, A. (2009). Cultivate self-efficacy for personal and organizational effectiveness. In E. A. Locke (Ed.). Handbook of principles of organization behavior. (2nd Ed., pp. 179-200). Oxford, UK: Blackwell.

Bernardo Carrasco, J. (2004). Una didáctica para hoy. Cómo enseñar mejor. Madrid: Rialp.

Bernardo Carrasco, J., Javaloyes Soto, J. J. y Calderero Fernández, J. F. (2008). Como personalizar la educación. Una solución de futuro. Madrid: Narcea.

Boekaerts, M. y Corno, L. (2005). Self-Regulation in the Classroom: A Perspective on Assessment and Intervention [Autorregulacion en el aula: Una perspectiva sobre su evaluacion e intervención]. Applied Psychology: An International Review, 54(2), 199-231.

Broc, M. A. (2011). Voluntad para estudiar, regulación del esfuerzo, gestión eficaz del tiempo y rendimiento académico en alumnos universitarios. Revista de Investigación Educativa, 29(1), 171-185. Recuperado de http:// revistas.um.es/rie/article/view/110731/126992

Campo, M. y Labarca, C. (2009). La teoría fundamentada en el estudio empírico de las representaciones sociales: Un caso sobre el rol orientador del docente. Opción. Revista de Ciencias Humanas y Sociales, 60. Recuperado de http://www.redalyc.org/articulo.oa?id=31012531004

Daura, F. T. (2013a). El contexto como factor del aprendizaje autorregulado en la educación superior. Educación y Educadores, 16(1), 109-125.

Daura, F. T. (2013b). Incidencia de las estrategias didácticas personalizadas en el desarrollo del aprendizaje autorregulado en estudiantes universitarios. Una experiencia en la carrera de Medicina (Tesis de doctorado inédita). Facultad de Filosofía y Letras. Universidad Nacional de Cuyo, Argentina.

Daura, F. T. (2014). Los docentes universitarios y su concepción sobre el aprendizaje, la autorregulación y la personalización educativa. Un análisis desde la teoría fundamentada. Forum Qualitative Sozialforschung / Forum: Qualitative Social Research, 15(2). Recuperado de http://www.qualitative-research.net/index.php/fqs/ rt/printerFriendly/2019/3663

Díaz-Barriga, Á. (2009). Pensar la didáctica. Buenos Aires: Amorrortu editores.

García Hoz, V. (1988a). Educación personalizada. Bogotá: Grupo editor Quinto Centenario.

García Hoz, V. (Dir.). (1988b). Tratado de la educación personalizada. La práctica de la educación personalizada. Madrid: Rialp.

Glaser, B. G. y Strauss, A. L. (1967). The discovery of Grounded Theory: strategies for qualitative research [El descubrimiento de la teoría fundamentada: Estrategias de investigación cualitativa]. Aldine: New York. 
Gregory, J. y Jones, R. (2009). Maintaining competence: A grounded theory typology of approaches to teaching in higher education [Mantenimiento de la competencia: Una tipología emergente sobre los enfoques de enseñanza en la educación superior]. Higher Education, 57(6), 769-785.

Hernández Sampieri, R., Fernández Collado, C. y Baptista Lucio, P. (1997). Metodología de la investigación. Colombia: Panamericana Formas e Impresos.

Hernández Sampieri, R., Fernández Collado, C. y Baptista Lucio, P. (2010). Metodología de la investigación. México: McGraw Hill.

Huber, G. L. (2008). Aprendizaje activo y metodologías educativas. Revista de Educación, Número extraordinario, 59-81.

Iglesias, J., Toscano, Ma. de la O. y Román, C. (2012). Taller de prensa económica como instrumento de innovación educativa. Revista de Investigación Educativa, 30(2), 423-440. Recuperado de http://revistas.um.es/rie/article/ view/137171/148901

Panadero, E. y Alonso Tapia, J. (2014). Teorías de autorregulación educativa: Una comparación y reflexión teórica. Psicología Educativa, 20(1), 11-22. Recuperado de http://pse.elsevier.es/es/teorias-autorregulacion-educativauna-comparacion/articulo/90326521/\#.U8bTK5R5OSp

Pérez, R. (2012). Sociedades multiculturales, interculturalidad y educación integral. La respuesta desde la educación personalizada. Revista Galega de Ensino, 13(45), 1-29. Recuperado de http://dialnet.unirioja.es/servlet/ articulo? codigo $=2555367$

Pintrich, P. R. (2000). The role of goal orientation in self-regulated learning. En M. Boekaerts; P. R. Pintrich y M. Zeidner (Eds.), Handbook of Self-Regulation [Manual de autorregulación, pp. 451-502]. San Diego: Academic Press.

Pintrich, P. R. (2002). The Role of Metacognitive Knowledge in Learning, Teaching, and Assessing [El rol del conocimiento metacognitivo en el aprendizaje, la docencia y la evaluación]. Theory into Practice, 41(4), 219-225.

Pintrich, P. R. y De Groot, E. V. (1990). Motivational and Self-Regulated Learning Components of Classroom Academic Performance [Componentes motivacionales y de aprendizaje autorregulado en el rendimiento académico]. Journal of Educational Psychology, 82(1). 33-40.

Pintrich, P. R., Smith, D., García, T. y McKeachie, W. (1991). A manual for the use of the Motivated Strategies for Learning Questionnaire (MSLQ) [Manual del cuestionario sobre estrategias motivacionales y de aprendizaje]. Michigan: National Center for Research to Improve Postsecondary Teaching and Learning. University of Michigan.

Rodríguez, J. L. (1996). Estrategias didácticas personalizadas en la Universidad. En García Hoz, V. (Dir.), La educación personalizada en la universidad, pp. 307-344. Madrid: Rialp.

Rué, J. (2009). El aprendizaje autónomo en educación superior. Madrid: Ed. Narcea.

Strauss, A. y Corbin, J. (1998). Fundamentos de investigación cualitativa. Técnicas y procedimientos para desarrollar teoría enraizada (M. E. González, A. M. Tello y V. González (Trads.). Mendoza: Facultad de Educación Elemental y Especial, UNCuyo.

Vasilachis de Gialdino, I. (Coord.). (2006). Estrategias de investigación cualitativa. Buenos Aires: Gedisa.

Zimmerman, B. J. (1989). A Social Cognitive View of Self-Regulated Academic Learning. [La vision sociocognitiva del aprendizaje autorregulado] Journal of Educational Psychology, 81(3), 329 -339.

\section{Notas}

[1] El presente trabajo forma parte de una investigación mayor desarrollada por la autora sobre la vinculación existente entre las estrategias didácticas personalizadas utilizadas en el aula universitaria y el desarrollo del aprendizaje autorregulado en estudiantes.

[3] Cuestionario de estrategias de motivación y de aprendizaje. 
Florencia Teresita Daura. Aprendizaje autorregulado e intervenciones docentes en la universidad [1...

[4] A tal efecto, los registros alcanzados en cada subcategoría se dividieron por la carga horaria total observada en las asignaturas. Por ejemplo, la subcategoría valor de la tarea se registró en 208 oportunidades, valor que se dividió por 169, que corresponde al total de horas observadas, obteniéndose 1,23 puntos.

[5] La cursiva es de la autora.

\section{BY-NC-ND}

